\title{
Improving the management of sepsis in a district general hospital by implementing the 'Sepsis Six' recommendations
}

\author{
Prashant Kumar, Mark Jordan, Jenny Caesar, Sarah Miller
}

South Canterbury DHB, New Zealand

\begin{abstract}
Sepsis is a common condition with a major global impact on healthcare resources and expenditure. The Surviving Sepsis Campaign has been vigorous in promoting internationally recognised pathways to improve the management of septic patients and decrease mortality. However, translating recommendations into practice is a challenging and complex task that requires a multi-faceted approach with sustained engagement from local stakeholders.
\end{abstract}

Whilst working at a district general hospital in New Zealand, we were concerned by the seemingly inconsistent management of septic patients, often leading to long delays in the initiation of life-saving measures such as antibiotic, fluid, and oxygen administration. In our hospital there were no clear systems, protocols or guidelines in place for identifying and managing septic patients.

We therefore launched the Sepsis Six resuscitation bundle of care in our hospital in an attempt to raise awareness amongst staff and improve the management of septic patients. We introduced a number of simple low-cost interventions that included educational sessions for junior doctors and nursing staff, as well as posters and modifications to phlebotomy trolleys that acted as visual reminders to implement the Sepsis Six bundle.

Overall, we found there to a be a steady improvement in the delivery of the Sepsis Six bundle in septic patients with $63 \%$ of patients receiving appropriate care within one hour, compared to $29 \%$ prior to our interventions. However this did not translate to an improvement in patient mortality.

This project forms part of an on going process to instigate a fundamental culture change among local healthcare professionals regarding the management of sepsis. Whilst we have demonstrated improved implementation of the Sepsis Six bundle, the key challenge remains to ensure that momentum of this project continues and forms a platform for sustainable clinical improvement in the long term.

\section{Problem}

Whilst working at a 130-bed district general hospital in New Zealand, we noted a high incidence of sepsis amongst patients both in the emergency department (ED) and general hospital wards. We were concerned by the seemingly inconsistent management of these patients, often leading to long delays in the initiation of lifesaving measures such as antibiotic, fluid, and oxygen administration. As part of a global effect to reduce mortality from sepsis, New Zealand endorses the Surviving Sepsis Campaign International Guidelines for the management of sepsis.[1] However, in our hospital there was no clear system in place for identifying septic patients and triggering a co-ordinated clinician response. Furthermore there were no local protocols or guidelines in place to aid in the delivery of optimal management for septic patients and subsequently the administration of antibiotics, intravenous fluids, and oxygen within one hour was poorly executed.

On further inspection, we felt that there were a number of factors that contribute to suboptimal implementation of international sepsis guidelines.[1] One was the lack of awareness and understanding amongst nurses and junior doctors that time-critical interventions significantly improves survival rates in septic patients.[1-5]
Furthermore, the assessment of end-organ perfusion with relevant blood tests, including lactate, to help determine the severity of sepsis and requirement for escalation of care to high dependency or intensive care units (ICU) was not performed well. One contributing factor to this phenomenon was the lack of a readily accessible time critical resuscitation bundle. Another was a lack of awareness amongst junior doctors regarding the necessary blood tests. This meant antibiotics were frequently delayed, sometimes for many hours, with blood cultures often acquired following administration of antibiotics.

\section{Background}

Sepsis is a common condition with a major global impact on healthcare resources and expenditure.[2] It is defined as the presence (probable or documented) of infection together with evidence of associated systemic manifestations, also known as systemic inflammatory response syndrome (SIRS).[1] Severe sepsis is defined as sepsis combined with sepsis-induced organ dysfunction or tissue hypoperfusion, whilst septic shock is defined as continued sepsis-induced hypotension despite adequate fluid resuscitation.[1] Data from studies conducted in mainly developed countries suggests that in adults, the incidence of severe sepsis is 
up to 300 cases per 100,000 population, and rising.[6-8] Reported in-hospital mortality for patients with severe sepsis or septic shock ranges between $20 \%$ and $50 \%$.[7,9-12] Meanwhile, data specific for Australasia demonstrates $23 \%$ in-hospital mortality rates for patients with severe sepsis or septic shock.[10] There are few disease processes with such a high mortality, with patients admitted with severe sepsis having a 6-10 fold higher mortality risk than if they presented with an acute myocardial infarction and a 4-5 times greater risk than if they had suffered an acute stroke.[2]

The fundamental approach to the management of sepsis includes early recognition, appropriate, and timely delivery of antibiotics, controlling the source of infection and adequate resuscitation with intravenous fluids and possibly vasoactive drugs.[10] Mortality has been shown to increase by $7.6 \%$ for every hour of delay in starting antibiotic therapy.[13] Unfortunately however, due to a variety of reasons including indecision by junior staff, availability of senior review and lack of awareness of the problem on general wards, there are frequently long delays between medical review and antibiotic administration. $[5,19]$ Meanwhile, whilst early goal-directed therapy (EGDT) has previously been associated with a $34 \%$ relative risk reduction in mortality, and was subsequently endorsed as a key strategy to reduce mortality from sepsis by the Surviving Sepsis Campaign, more recent studies have not shown any benefit in terms of all-cause mortality in patients with early septic shock.[1,3,10]

The Surviving Sepsis Campaign is a joint collaboration of the Society of Critical Care Medicine and the European Society of Intensive Care Medicine committed to reducing mortality from sepsis worldwide.[1] This campaign included recommendations concerning the implementation of a core set of evidence-based interventions, otherwise known as 'resuscitation bundles'.[1] Use of such bundles has been associated with a decrease in mortality.[15] One such bundle is 'Sepsis Six' which was specifically designed to facilitate early intervention in busy hospital and pre-hospital settings. $[5,16]$ Delivery of the Sepsis Six resuscitation bundle is associated with a $55 \%$ relative risk reduction in mortality.[17] Previous quality improvement projects reported in this journal, have demonstrated implementation of the Sepsis Six bundle through a variety of different interventions within different hospital settings, to varying degrees of success.[18-20] What is consistent throughout these projects however, is the recognition that successfully implementing the bundle is a challenging and complex task that requires a multi-faceted approach with consistent engagement from local stakeholders.[18-20]

The Sepsis Six is comprised of three diagnostic and monitoring steps and three therapeutic interventions:

1. Deliver high-flow oxygen

2. Take blood cultures prior to antibiotics but do not delay treatment

3. Administer empirical intravenous antibiotics

4. Measure serum lactate

5. Start intravenous fluid resuscitation with crystalloids

6. Commence urine output monitoring via either a catheter or chart[5]
Reliable, timely delivery of more complex life-saving tasks, such as EGDT, demands greater awareness, faster recognition and initiation of basic care, and more effective collaboration between clinicians and nurses involved in the initial assessment of the septic patient. As a life threatening condition associated with a high rate of mortality, quality care of patients with sepsis is paramount. Due to concerns regarding early assessment and initiation of appropriate therapy previously identified, we set about introducing the Sepsis Six resuscitation bundle into our hospital in an attempt to deliver appropriate care in a timely manner to septic patients and thereby improve their outcomes.

\section{Baseline measurement}

Data was retrospectively collected over a six-month period for all hospitalised adult (over 18-years old) patients with confirmed sepsis. Initially, case notes were obtained via the medical records department, using a list of coded diagnoses that included infection, sepsis, pneumonia, lower and upper respiratory infection, urosepsis, urinary tract infection, pyrexia of unknown origin, meningitis, abdominal sepsis, biliary sepsis, bacteraemia, septicaemia, endocarditis, pyelonephritis, septic arthritis, and cellulitis. 138 sets of notes were then scrutinised to identify patients that satisfied sepsis or severe sepsis criteria as defined by the international sepsis guidelines shown below.[1]

Sepsis- clinical evidence of infection and any two of the following present;

1. Temperature $<36^{\circ} \mathrm{C}$ or $>38^{\circ} \mathrm{C}$

2. Respiratory rate $>20 / \mathrm{min}$ or pCO $2<4.3 \mathrm{kPa}(<32 \mathrm{mmHg})$

3. White cell count $<4 \times 10^{\wedge} \mathrm{g} / \mathrm{L}$ or $>12 \times 10^{\wedge} 9 / \mathrm{L}$

4. Heart rate $>90 \mathrm{bpm}$

Severe sepsis- patients with sepsis plus and any one of the following present:

1. Systolic BP $<90 \mathrm{mmHg}$

2. Mean arterial pressure $<65 \mathrm{mmHg}$

3. Lactate $>2 \mathrm{mmol} / \mathrm{L}$

4. Other evidence of organ dysfunction: (creatinine $>177$ umol/L, bilirubin $>34$ umol/L, platelets $<100 \times 10^{\wedge} 9 / \mathrm{L}$, INR $>1.5$, urine output $<0.5 \mathrm{~mL} / \mathrm{kg} / \mathrm{hr}, \mathrm{SpO} 2<90 \%$ )

Those who met the criteria for sepsis or severe sepsis were subsequently included in the data collection. Our primary outcome measure was administration of antibiotics, intravenous fluids, and oxygen if appropriate within one hour of initial assessment. Secondary outcome measures focused on fulfilling the Sepsis Six bundle that included obtaining blood cultures prior to antibiotic administration, lactate levels, and documentation of urine output monitoring either via indwelling catheter or fluid balance chart. Furthermore we analysed data regarding time taken from initial nursing assessment to clinician review and all-cause in-hospital mortality.

A total of 55 patients (26 males, 29 females) were included in the baseline measurement. 22 patients met the criteria for severe 
sepsis. 16 out of 55 patients (29\%) had appropriate management with antibiotics, intravenous fluids +/- oxygen commenced within 1 hour. Only 10 out of 55 patients (18\%) had lactate measured. 18 (33\%) had blood cultures taken and of this group 14 (78\%) were taken prior to antibiotic administration. 15 out of 55 patients $(27 \%)$ had urine output monitoring via either an indwelling catheter or designated fluid input/output chart. After initial assessment by nursing staff, the mean waiting time to clinician review was 47 minutes (range 0-270). One patient, who did not fit criteria for severe sepsis at initial assessment, died whilst in hospital (2\%).

Overall, the numbers of patients receiving the equivalent of a sepsis bundle were as follows:

Six parts: $2(4 \%)$

Five parts: $6(11 \%)$

Four parts: $11(20 \%)$

Three parts: $11(20 \%)$

Two parts: $13(24 \%)$

One part: $10(18 \%)$

Zero parts: 2 (4\%)

\section{Design}

When considering the underlying causes of the problem, it became obvious that a multi-faceted set of interventions were necessary to improve the management of sepsis in our hospital. Firstly, since there was no readily available and accessible sepsis resuscitation bundle in place, we introduced a Sepsis Six resuscitation bundle algorithm which was reproduced in poster format and displayed in relevant areas of the hospital such as the ED, general ward care stations and doctors' offices. They were intended to act as a visual reminder to staff to implement the six appropriate diagnostic and therapeutic steps of the algorithm, ideally within one hour of initial assessment.

Secondly, we set out to engage key stakeholders to improve awareness of the implications of poor management of sepsis and the strategies we could employ to improve the situation. This was achieved through a variety of presentations, workshops, and informal discussions with both junior and senior medical and nursing staff. These were conducted over a two-week period and focused on ED and general ward staff. We presented the Sepsis Six resuscitation bundle and used data from our baseline measurement as a rationale for adopting these modifications to practice, thereby attempting to foster a fundamental culture change from the hospital staff who, up until this point, were not familiar with using resuscitation bundles as tools to improve the management of septic patients. These sessions focused on the recognition, investigation, monitoring, and management of septic patients and the importance of Sepsis Six implementation in improving patient outcomes and reducing mortality.
Finally, we designated a number of 'sepsis assistants' throughout the general wards and ED to ensure that education regarding the management of sepsis was reinforced on a day-to-day basis, and that the momentum of our project was sustained. These 'assistants' were able to aid staff and advise on appropriate initiation and escalation of treatment, finding equipment and requesting appropriate investigations.

As noted previously, sustainability of such projects is a difficult challenge, often due to the high turn around of staff, especially junior doctors who consistently rotate through different jobs in different hospitals.[18-19] We were especially keen to ensure that improvements we made in our hospital were sustainable in the long term. We therefore recruited junior doctors to the sepsismanagement quality improvement team, who we knew would be working in the hospital for the next two years. This was to ensure that the momentum of the project was sustained, even once some members of the team moved on to different jobs. Recruitment of future members of the team will follow a similar practice to ensure that progress is continued.

\section{Strategy}

PDSA cycle 1: Meetings were held by the sepsis-management quality improvement team to review national and international sepsis guidelines and evaluate how they could be implemented within our hospital. A sepsis recognition and management pathway was then developed. Our aim was that this pathway, based on the Sepsis Six,[5] could be used to prompt the consideration of sepsis in unwell patients and to guide the user through the diagnostic and therapeutic steps required in the management of the septic patient (intravenous fluids, blood cultures, antibiotics, lactate, oxygen, monitor urine output).

PDSA cycle 2: To raise awareness of sepsis diagnostic criteria and the Sepsis Six pathway, we designed sepsis recognition and management algorithm posters for display in the ED and all wardbased doctors' offices and nursing care stations. A first draft was displayed in the ED and received positive feedback from staff detailing that it was easy to understand and clearly highlighted the Sepsis Six resuscitation bundle. The design was bold and colourful with an easy-to-read font. After minor layout adjustments, the poster was distributed to the wards and received promising feedback from stakeholders.

PDSA cycle 3: We held an educational awareness workshop on sepsis with all ED staff and all hospital junior doctors invited to attend. $75 \%$ of ED staff and $30 \%$ of junior doctors attended the session. The session focused on the early recognition and management of sepsis and was designed to emphasise the importance of recognising sepsis and the timely initiation of the Sepsis Six bundle to improve patient outcomes. Examples of clinical scenarios were used to give realistic representations of septic patients and help contextualize background theory into clinical practice. Feedback from the session demonstrated that junior nurses and doctors, in particular, found the workshop extremely useful. We therefore repeated the workshop at one of the weekly junior doctor teaching sessions. This was attended by just 
over $65 \%$ of junior doctors. We also held workshops in each of the general wards, although only $30 \%$ of nurses were able to attend due to clinical duties. We therefore designated a number of 'sepsis assistants' throughout the general wards and the ED to ensure that sepsis education could be continued and reinforced on a day-to-day basis in a more informal environment.

PDSA cycle 4: Three months following implementation of these interventions, we conducted a re-audit. Whilst the results were promising, further improvements could still be made. We noted that the vast majority of patients with sepsis presented to the ED, with the number of ward-based patients that become septic comparatively low. We therefore focussed our next set of interventions in the ED and sought feedback from ED staff as to how to improve implementation of the Sepsis Six bundle. Nursing staff commented how they would often forget to take lactate and blood cultures from patients who whilst tachypnoeic and tachycardic, were afebrile during the initial assessments. We therefore tied a laminated photo of a set of blood cultures, a lactate tube, nasal prongs, a $500 \mathrm{ml}$ bag of normal saline, a catheter, and vial of antibiotic on each phlebotomy trolley in the department. Feedback from the nurses was extremely positive, remarking that this photo reminded them to draw the relevant blood samples and consider escalating management of possibly septic patients by triaging them higher for a more urgent clinician review.

PDSA cycle 5: We noted that there was no change in mean waiting time to clinician review from initial nursing assessment. ED nursing staff reported that even if patients were tachypnoeic, tachycardic, and febrile, and therefore categorised as a higher triage priority, due to staff shortages in the ED, there were still delays in clinician reviews. This was highlighted as a critical incident, and brought up in a series of departmental meetings. As a consequence, another full-time consultant is being recruited to the ED, to help improve timely reviews of all patients, including those who may be septic.

PDSA cycle 6: Following another three months, a re-audit demonstrated further significant improvements in the implementation of the Sepsis Six bundle. These results are due to be presented at a hospital managers meeting, to gain formal approval for the integration of the Sepsis Six bundle into hospital guidelines. These will be placed on the trust intranet and be readily accessible to any healthcare professional in the hospital. Furthermore, we are extending the sepsis management quality improvement team to incorporate a wider range of stakeholders including the antimicrobial pharmacist, the resident microbiologist and the ED clinical nurse manager. We are also working on introducing 'sepsis boxes' in the general wards to centralise the equipment needed to adequately manage septic patients. This has previously been shown to improve implementation of the Sepsis Six bundle.[18] Finally, we are incorporating sepsis management education sessions into the induction programme for newly appointed doctors. Further re-audit will be completed in three months time to re-evaluate our practice, following which the sepsis management quality improvement team will meet once more to discuss the future direction of the project.
3 months post baseline measurement: A total of 71 patients (34 males, 37 females) were included in the data collection. 22 patients met the criteria for severe sepsis. 37 out of 71 patients $(52 \%)$ had appropriate management with antibiotics, intravenous fluids $+/$ oxygen commenced within 1 hour. Only 7 out of 71 patients (10\%) had lactate measured. 37 (52\%) had blood cultures taken and of this group all $37(100 \%)$ were taken prior to antibiotic administration. 26 out of 71 patients (37\%) had urine output monitoring via either an indwelling catheter or designated fluid input/output chart. After initial assessment by nursing staff, the mean waiting time to clinician review was 48 minutes (range 0-345). 9 out of 71 patients (13\%) died whilst in hospital, with 5 out of these 9 patients fitting criteria for severe sepsis at initial assessment.

Overall, the numbers of patients receiving the equivalent of a sepsis bundle were as follows:

Six parts: $6(8 \%)$

Five parts: $15(21 \%)$

Four parts: $14(20 \%)$

Three parts: $17(24 \%)$

Two parts: $13(18 \%)$

One part: $6(8 \%)$

Zero parts: $0(0 \%)$

6 months post baseline measurement: A total of 40 patients (23 males, 17 females) were included in the data collection. 17 patients met the criteria for severe sepsis. 25 out of 40 patients (63\%) had appropriate management with antibiotics, intravenous fluids $+/-$ oxygen commenced within 1 hour. Only 10 out of 40 patients (25\%) had lactate measured. 25 (63\%) had blood cultures taken and of this group $24(96 \%)$ were taken prior to antibiotic administration. 17 out of 40 patients (43\%) had urine output monitoring via either an indwelling catheter or designated fluid input/output chart. After initial assessment by nursing staff, the mean waiting time to clinician review was 44 minutes (range $0-205)$. 4 out of 40 patients (10\%) died whilst in hospital, with all 4 patients fitting criteria for severe sepsis at initial assessment.

Overall, the numbers of patients receiving the equivalent of a sepsis bundle were as follows:

Six parts: 5 (13\%)

Five parts: $10(25 \%)$

Four parts: 11 (28\%)

Three parts: 7 (18\%)

Two parts: 4 (10\%)

\section{Results}


Overall, we saw a significant improvement in the implementation of the Sepsis Six bundle, with $66 \%$ of patients receiving 4-6 parts compared to $35 \%$ at baseline. However these improvements did not translate to better patient outcomes, and indeed we noted worsening in-hospital mortality since the start of our project, from $2 \%$ to $10 \%$. This may reflect the fact that our re-audits were completed during the winter when patients are generally more unwell. To further evaluate these trends we will need to compare future data sets from comparable times of the year. Furthermore, there was no significant decrease in time taken from initial nursing assessment to clinician review, and this may be partly explained by the fact that the new ED consultant post has not been filled as yet.

See supplementary file: ds6046.doc - "Sepsis Six Data"

\section{Lessons and limitations}

We have learnt a number of important lessons during this project, none more so than the importance of a multi-faceted approach to tackling an inherently complex problem. Certainly there is no magic answer to improving the management of sepsis in busy clinical environments. It requires a co-ordinated effort from a dedicated team to instigate changes, and even then, progress may prove to be slow. However, small changes can lead to improvements in practice, and this project highlights the need for healthcare professionals to be continually motivated to achieve such improvements.

Another key lesson is that a singular approach does not necessarily work in different situations or departments, and therefore flexibility is vital to achieving success. For example, we broached the idea of introducing a 'sepsis box' or 'sepsis trolley' into the ED. These have been successfully trialled in previous quality improvement projects.[18-20] However, due to limited space, this idea was thought to be impractical by department managers and we modified our intervention accordingly. The original concept however, may still help in managing septic patients on the general wards, and we are therefore implementing this idea during the next cycle of interventions.

Admittedly there are a number of limitations to this project. Firstly this study was conducted over a six-month period, and as such its sustainability is, as yet, untested. Therefore, the key challenge is to ensure the sustainability of this project for the future. To accomplish this, we needed to extend the number of stakeholders involved in the team. Initially this was a junior doctor-led initiative, as it was felt that to kick-start the project it would be better to have a smaller but highly motivated and dedicated team. Now the project is up and running and there is greater awareness across the hospital, we hope that with more of a multidisciplinary approach the improvements that we have made so far can be consolidated and further improved upon, and that these may in turn lead to better outcomes for our patients. Additionally, involvement of the other members of the multidisciplinary team may bring new ideas to the
Another key limitation of this study is that it does not examine the costs associated with managing sepsis according to the Sepsis Six bundle. Nor does it explore any savings that may occur by reducing the incidence of septic patients becoming more unwell. Whilst this would be difficult to incorporate into the study, it will be discussed at future meetings.

Finally, the lack of outcome measures in our data analysis is another limitation of this study. For example, we did not analyse ICU admission rates, use of vasoactive drugs, appropriate antibiotic stewardship, length of stay in hospital, or post-discharge morbidity and mortality. By increasing the stakeholders involved in the project, we hope to increase the content of the data we can collect which will provide us with more information to guide future practice.

\section{Conclusion}

As noted previously, sepsis is a serious condition with high mortality. The international community has been vigorous in promoting internationally recognised pathways to improve the management of septic patients in an attempt to improve their outcomes. However, translating recommendations into practice is a challenging and complex task that requires a multi-faceted approach with sustained engagement from local stakeholders. We have implemented a number of simple, low-cost interventions that have improved the implementation of the Sepsis Six resuscitation bundle in our hospital. However, this has not translated into better patient outcomes in terms of mortality. Whilst this quality improvement project has helped instigate a fundamental culture change among local healthcare professionals, there is more work to be done to improve the management of sepsis at our hospital. The key challenge remains to ensure that momentum of this project continues and forms a platform for sustainable clinical improvement in the long term.

\section{References}

1. Dellinger RP, Levy MM, Rhodes A, et al. Surviving sepsis campaign: International guidelines for the management of severe sepsis and septic shock: 2012. Crit Care Med 2013;41(2):580-637

2. Daniels, R. Surviving the first hours in sepsis: getting the basics right (an intensivist's perspective). J Antimicrob Chemother. 2011;66(suppl 2):ii11-23

3. Rivers E, Nguyen B, Havstad S, et al. Early goal-directed therapy in the treatment of severe sepsis and septic shock. N Engl J Med 2001;345:1368-77.

4. Baxter S, Hutchings $S$, \& Barnes T. Timeliness of antibiotic administration in septic shock. In: Colvin JR, \& Pedan CJ. [3rd ed.] Raising the standard: a compendium of audit recipes for continuous quality improvement in anaesthesia. London: Royal College of anaesthetists; 2012. Available from www.rcoa.ac.uk/system/files/CSQ-ARB2012-PRELIM.pdf

5. Robson WP, \& Daniels R. The Sepsis Six: helping patients 
to survive sepsis. Br J Nurs 2008;17:16-21

6. Angus DC, Linde-Zwirble WT, Lidicker J, Clermont G, Carcillo J, \& Pinsky MR. Epidemiology of severe sepsis in the United States: analysis of incidence, outcome, and associated costs of care. Crit Care Med 2001;29:1303-10

7. Gaieski DF, Edwards JM, Kallan MJ, \& Carr BG. Benchmarking the incidence and mortality of severe sepsis in the United States. Crit Care Med 2013;41:1167-74

8. Jawad I, Luksic I, \& Rafnsson SB. Assessing available information on the burden of sepsis: global estimates of incidence, prevalence and mortality. J Glob Health 2012;2(1):010404

9. Australasian Resuscitation in Sepsis Evaluation (ARISE) Investigators and the Australian and New Zealand Intensive Care Society (ANZICS) Adult Patient Database (APD) Management Committee. The outcome of patients with sepsis and septic shock presenting to emergency departments in Australia and New Zealand. Crit Care Resusc 2007;9:8-18.

10. Peake SL, Delaney A, Bailey M, et al. Goal-directed resuscitation for patients with early septic shock. N Engl J Med 2014;371:1496-1506

11. Quenot JP, Binquet C, Kara F, et al. The epidemiology of septic shock in French intensive care units: the prospective multicentre cohort EPISS study. Crit Care 2013;17:R65

12. Yealy DM, Kellum JA, Huang DT, et al. A randomized trial of protocol-based care for early septic shock. N Engl J Med 2014;370:1683-1693

13. Kumar A, Roberts D, Wood KE, et al. Duration of hypotension before initiation of effective antimicrobial therapy is the critical determinant of survival in human septic shock. Critical Care Medicine 2006; 34:1589-1596.

14. Marwick CA, Guthrie B, Pringle JE, et al. A Multifaceted intervention to improve sepsis management in general hospital wards with evaluation using segmented regression of interrupted time series. British Medical Journal 2013;0:1-8

15. Levy MM, Dellinger RP, Townsend SR, et al. The Surviving Sepsis Campaign: results of an international guidelinebased performance improvement programme targeting severe sepsis. Crit Care Med 2010;38:1-8

16. Robson W, Nutbeam T, \& Daniels R. Sepsis- a need for prehospital intervention? Emerg Med J 2009;26:535-5388.

17. Daniels R, Nutbeam T, McNamara G, Galvin C. The sepsis six and the severe sepsis resuscitation bundle: a prospective observational cohort study. EMJ $2011 ; 28(6): 507-12$

18. Kafle $\mathrm{S}$, \& Nath N. Improving management of severe sepsis and uptake of sepsis resuscitation bundle in an acute setting. BMJ Qual Improv Report 2014;u204152.w1807 doi: 10.1136/bmjquality.u204152.w1807

19. Adcroft L. Improving sepsis management in the acute admissions unit. BMJ Qual Improv Report 2014;u204974.w2091 doi: 10.1136/bmjquality.u204974.w2091

20. McGregor C. Improving time to antibiotics and implementing the "Sepsis 6". BMJ Qual Improv Report 2014;u202548.w1443 doi: 10.1136/bmjquality.u202548.w1443

\section{Declaration of interests}

Nothing to declare

\section{Acknowledgements}

Elaine Clark

Rachel Mills

Raewyn Brown

Ethical approval

Local policy did not require ethical approval to be sought for this project 